\title{
Design of a hybrid refractive-diffractive telescope for observations in UV
}

\section{Grzegorz Fluder ${ }^{1,2}$ (1)}

Received: 9 January 2020 / Accepted: 10 July 2020 / Published online: 19 July 2020

(C) The Author(s) 2020

\begin{abstract}
Telescopes are one of the common types of satellite payloads. They are used both for Earth and astronomical observations. By using space telescopes it is possible to eliminate the negative effect of the atmosphere on image quality. Additionally, observations in some spectral ranges can be performed only from space due to absorption of certain wavelengths in the atmosphere. One such range is UV below $300 \mathrm{~nm}$, which is of particular interest when it comes to the investigation of hot objects. Reflective telescopes are commonly used in this spectral range, although many classical designs are limited in their useful field of view to values below $1^{\circ}$. In this paper a hybrid refractive-diffractive telescope design working in a $200 \mathrm{~nm}-300 \mathrm{~nm}$ spectral range with a field of view $10^{\circ} \times 10^{\circ}$ is proposed. Its performance is compared to purely refractive and reflective systems and significant improvement in the imaging quality of the system and decrease of its size is shown. The choice of the diffractive element type is explained. Parameters of the systems are based on the requirements for a proposed Polish mission UVSat which aims to enable long-term observations of a large number of stars exhibiting UV variance.
\end{abstract}

Keywords Telescope $\cdot$ Hybrid imaging system $\cdot$ Space telescope $\cdot$ Diffractive optical element $\cdot$ Ultraviolet astronomy

\section{Introduction}

Ultraviolet astronomy offers unique possibilities to investigate hot object, such as young and massive stars, stars at the final stages of their evolution and others, such as active galactic nuclei. Due to the high absorption of Earth's atmosphere in this

\section{Grzegorz Fluder}

g.fluder@ solarisoptics.eu

1 Solaris Optics S.A, Sobieskiego 49, 05-410 Józefów, Poland

2 Institute of Micromechanics and Photonics, Photonics Engineering Division, Warsaw University of Technology, 8 Św. A. Boboli St, 02-525 Warsaw, Poland 
spectral range observations may be performed only from space. There are several satellite missions currently being realized or developed offering the possibility of UV observations. They include among others World Space Observatory - Ultraviolet [1] Astrosat with its Ultraviolet Imaging Telescope (UVIT) [2], Large Ultraviolet Optical Infrared Surveyor (LUVOIR) [3] and Hubble Space Telescope with several instruments, including the Space Telescope Imaging Spectrograph [4] The concept of a Polish satellite mission UVSat has been first proposed in 2013 aiming to enable longterm observations of even several hundred objects simultaneously [5]. Thanks to this unique feature a very wide range of objects exhibiting UV variance may be observed and analyzed. Depending on the object this variance could last from single seconds even up to years. All the data gathered during such observations could lead to development and verification of different models, e.g. in the field of asteroseismology. The most important classes of objects to be observed include pulsating main sequence stars, hot and massive stars, binary stars and the early stages of supernovae. More detailed concept and analysis of the mission has been presented in the feasibility study 'Astronomical Observation Satellite in UV; Pre-feasibility study for UVSat satellite' prepared in 2016 for Polish Space Agency. In 2020 a project proposal will be submitted to the funding agency with the plan to launch the satellite by the end of 2023 .

During the design and manufacturing of telescopes working in ultraviolet range several important issues have to be considered. One of them is the limited selection of optical materials with high transmission in this spectral range [6]. Available materials, such as fused silica, calcium fluoride or magnesium fluoride exhibit significant dispersion in ultraviolet. As an example, for magnesium fluoride the difference between refractive index values for wavelengths $200 \mathrm{~nm}$ and $300 \mathrm{~nm}$ is almost twice as large as for $300 \mathrm{~nm}$ and $700 \mathrm{~nm}$. Due to this fact, single lenses introduce larger chromatic aberration in UV compared to the ones working in the visible range. Additionally, available materials have relatively similar refractive indices and Abbe numbers, making it difficult to correct chromatic aberration. In refractive telescopes working in UV, to correct those aberrations, it is often required to use more lenses compared to telescopes with similar parameters working in the visible range. In the case of space telescopes it increases not only manufacturing costs, but also due to the higher weight the launch costs are larger. Ultraviolet telescopes are also demanding from a manufacturing point of view, since surface form errors and microroughness of the optical surfaces deteriorates the performance of the system more significantly for shorter wavelengths.

\section{Use of diffractive optical elements in imaging systems}

Diffractive optical elements (DOEs) offer unique possibilities when it comes to aberration correction. Their important feature is dispersion. Refractive elements introduce positive chromatic aberration, meaning that their focal length decreases with the increase of wavelength. DOEs on the other hand have negative dispersion. Figure 1 illustrates this property of refractive and diffractive elements. Taking into account that property, refractive and diffractive elements may be combined to correct chromatic aberration [7]. 


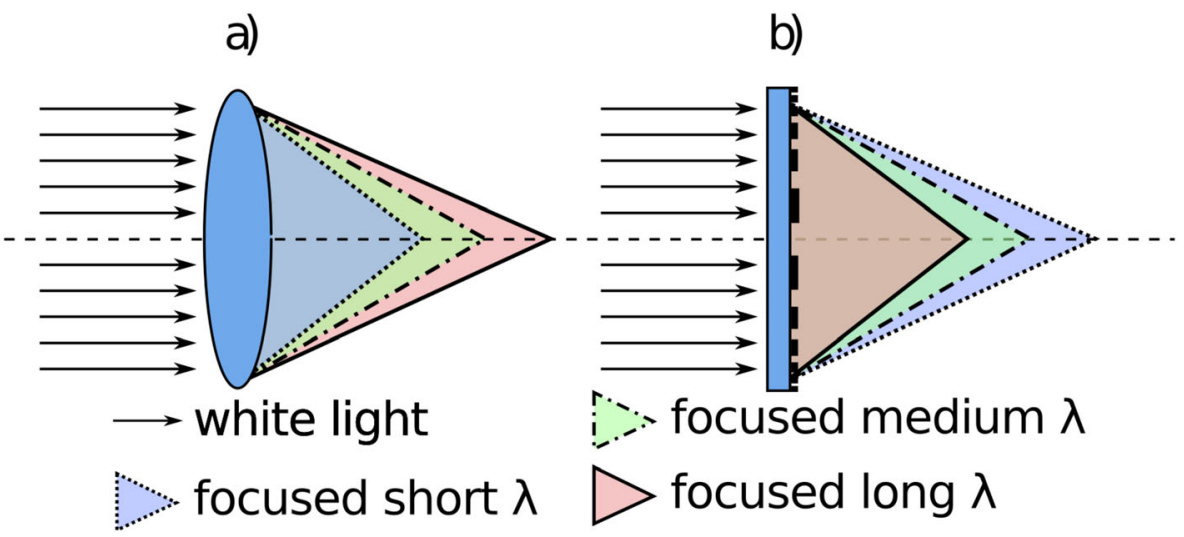

Fig. 1 Chromatic aberration of a) refractive element, b) diffractive element

Another important feature of DOEs is that arbitrary phase function may be introduced without significant impact on their manufacturability and measurability. Desired phase profile is obtained by proper choice of zone widths. Methods such as e-beam or UV lithography make it possible to manufacture structures with feature widths in the order of a fraction of micrometer, which gives large flexibility when it comes to optimizing diffractive elements. By the proper design of zone widths one can easily obtain "aspherical" diffractive lenses, making it possible to not only correct chromatic, but also monochromatic aberrations. This can lead to the reduction of the mass and the number of elements in the optical system.

\section{Design of the optical systems}

At the early stage of the UVSat project the following requirements for the telescope have been proposed [5]:

- Central wavelength equal to $250 \mathrm{~nm}$ and bandwidth up to $100 \mathrm{~nm}$;

- Entrance pupil diameter 50-120 mm;

- Angular field of view $10^{\circ} \times 10^{\circ}$;

- Focal length resulting in angular resolution 10"-20"/pixel;

- Pixel size $10 \mu \mathrm{m}$.

In the next subsections designs of three different telescopes fulfilling above requirements are presented.

\subsection{Reflective telescope}

Due to the relatively large required field of view the most common types of reflective telescopes, such as Ritchey-Chretien, could not be used. Instead, a three-mirror unobscured system has been designed following the principles outlined by Hallam et al. [8]. The entrance pupil of the system was set to be equal to $50 \mathrm{~mm}$, which is the lowest acceptable value, and the obtained focal length is equal to $150 \mathrm{~mm}$, resulting in 
f-number 3. The first surface is convex spherical, the second one is concave prolate elliptical and the third mirror has a concave spherical surface. The diameter of the largest, second, mirror is equal to $152 \mathrm{~mm}$. The aspherical surface has a maximal deviation from the best fit sphere equal to $23.7 \mu \mathrm{m}$. All three mirrors are offset segments of their parent mirrors which have axes and vortices lie on system's optical axis. Figure 2 shows the layout of the system and its performance: spot diagrams, encircled energy and MTF (Modulation Transfer Function) curves. The most prevalent aberration is field curvature. Since the system does not have a rotational symmetry, performance of the system is different for positive and negative field angle values.

The obtained system is large with a $530 \mathrm{~mm}$ separation between the secondary and tertiary mirror. The mass of the mirrors is equal to $1350 \mathrm{~g}$ with fused silica as substrate material and $1550 \mathrm{~g}$ for Zerodur. Despite the obvious advantages of purely reflective systems, such as no chromatic aberration and no absorption in the materials, the size of the designed telescope makes it an impractical solution to this particular task.

\subsection{Refractive system}

Designed purely refractive telescope consists of eight elements. It is a modified Petzvaltype objective with additional field flattener. In Fig. 3 the layout and performance of the system is shown. Lenses 1, 3, 4 and 5 are made of $\mathrm{MgF}_{2}$, lenses 2, 6 and 8 of Fused Silica and element 7 of $\mathrm{CaF}_{2}$. The entrance pupil diameter is equal to $90 \mathrm{~mm}$ and the focal length for $250 \mathrm{~nm}$ wavelength is $150 \mathrm{~mm}$ (f-number 1.67). The total track length

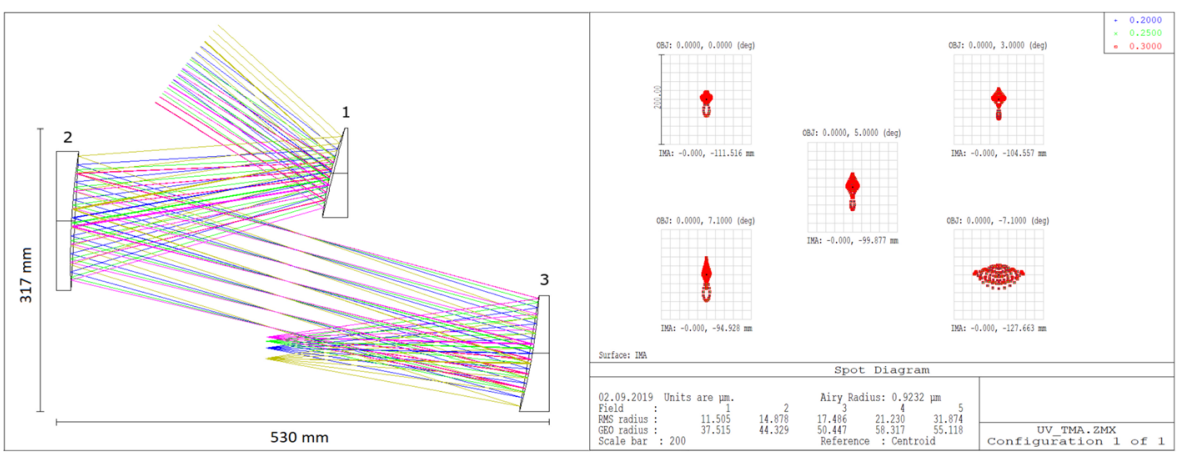

a)

b)

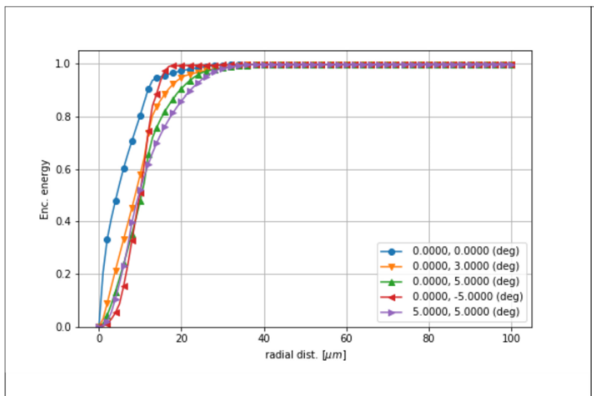

c)

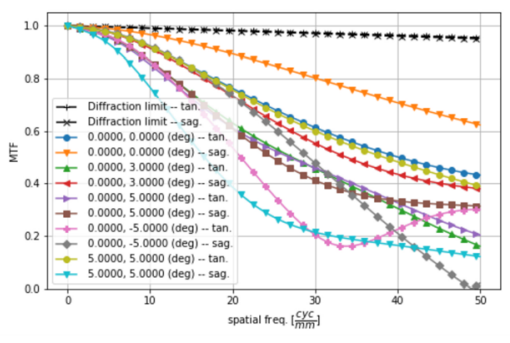

d)

Fig. 2 Scheme and performance plots for reflective system. a) optical layout of the telescope, b) spot diagrams, c) geometric encircled energy, d) MTF curves 
from the first lens surface to the image plane is equal to $234 \mathrm{~mm}$. The aperture stop is located between lenses 3 and 4 . The system is significantly smaller than the reflector. The total mass of the lenses is equal to $1630 \mathrm{~g}$ making them heavier than the mirrors of the reflective telescope, however the aperture of the refractor is significantly larger. It was not possible to fully correct the aberrations in this system, for each field value used in the optimization $40 \%$ of energy is confined within a $40 \mu \mathrm{m}$ circle.

\subsection{Hybrid system}

Hybrid refractive-diffractive system with the same parameters as the refractive system has been designed. It consists of 7 lenses with the first one having a diffractive structure on its second, flat surface. Figure 4 summarizes the details of designed system. The mass of the lenses is equal to $1500 \mathrm{~g}$. The DOE is designed in such a way that -1 diffraction order is used for image formation. In geometrical ray tracing only zone widths of the diffractive structure are important, as it is the only parameter influencing diffraction angles. $100 \%$ efficiency in chosen diffraction order is assumed by the software. The system has one fewer lens compared to the purely refractive system. In this system, depending on field value, between $88 \%$ and $100 \%$ of energy is confined within the $40 \mu \mathrm{m}$ spot for every object point. Comparing it to $40 \%$ obtained for the same diameter in refractive system, one can observe a very significant improvement in the performance of the system.

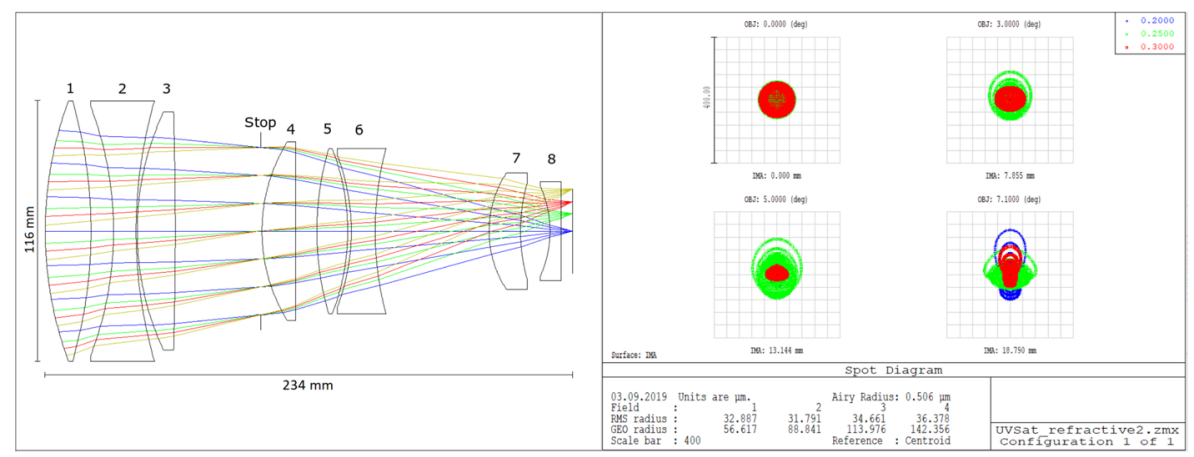

a)

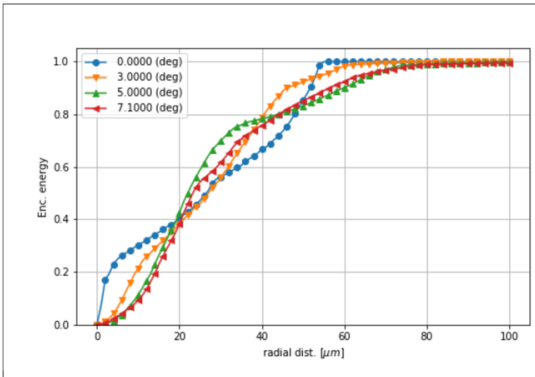

c) b)

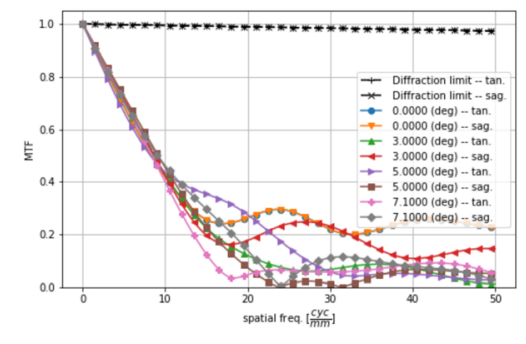

d)

Fig. 3 Scheme and performance plots for refractive system. a) optical layout of the telescope, b) spot diagrams, c) geometric encircled energy, d) MTF curves 


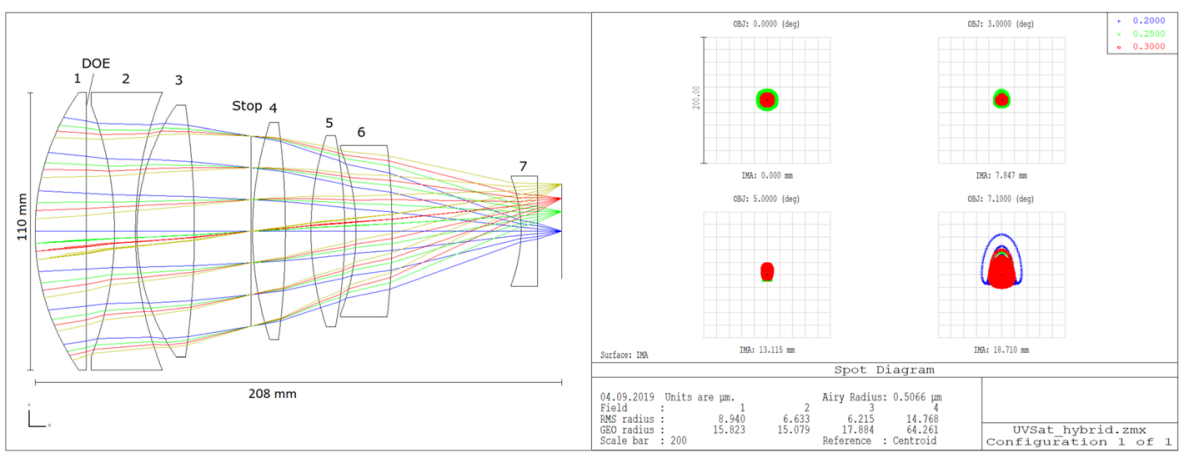

a)

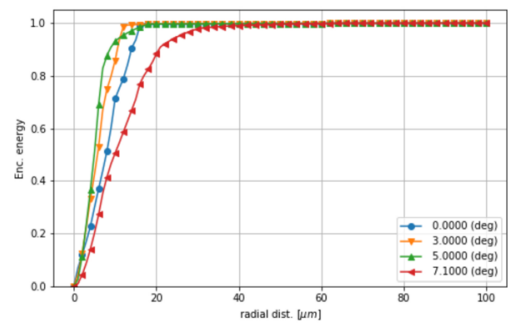

c) b)

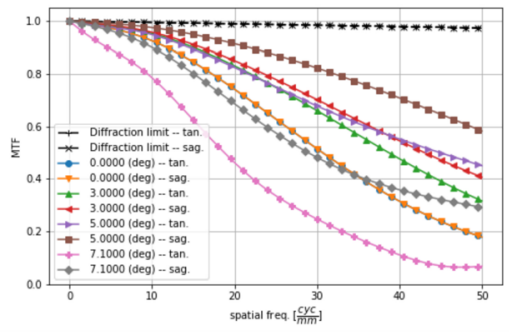

d)

Fig. 4 Scheme and performance plots for hybrid refractive-diffractive system. a) optical layout of the telescope, b) spot diagrams, c) geometric encircled energy, d) MTF curves

\subsection{Summary of the designs}

In Table 1 a comparison of the parameters of the three designed systems is presented. It can be seen that reflective and hybrid systems offer similar level of aberration correction, however the hybrid system has significantly larger aperture. It is also much shorter than the reflector, making it more suitable for use in small satellites. Refractor offers the worst imaging quality of the designed systems.

Table 1 Summary of the parameters of the systems

\begin{tabular}{|c|c|c|c|}
\hline & Reflective & Refractive & Hybrid \\
\hline Focal length $[\mathrm{mm}]$ & 150 & 150 & 150 \\
\hline Entrance pupil diameter $[\mathrm{mm}]$ & 50 & 90 & 90 \\
\hline Length of the system [mm] & 530 & 234 & 208 \\
\hline Mass of elements $[\mathrm{g}]$ & 1350(Fused Silica)/1550 (Zerodur) & 1630 & 1500 \\
\hline Number of elements & 3 & 8 & 7 \\
\hline RMS spot diameter on axis $[\mu \mathrm{m}]$ & 16.1 & 65.8 & 17.9 \\
\hline RMS spot diameter in full field $[\mu \mathrm{m}]$ & 28 & 72.8 & 29.5 \\
\hline Energy encircled in a $40 \mu \mathrm{m}$ spot on axis [\%] & 98 & 40 & 100 \\
\hline Energy encircled in a $40 \mu \mathrm{m}$ spot in full field [\%] & 86 & 40 & 88 \\
\hline
\end{tabular}




\section{Choice of DOE type}

There are two types of diffractive optical elements which are commonly used in imaging systems - single- (SLDOE) and multilayer (MLDOE) diffractive optical elements. SLDOE is a single diffractive structure with a constant or variable local period. This type of element is shown in Fig. 1 b). Usually they have a uniform profile depth across the whole surface, calculated to obtain high diffraction efficiency in the desired order for one wavelength. For other wavelengths efficiency is lower, resulting in more energy being spread to different, undesired diffraction orders. It can be calculated using the formula [7]:

$$
\eta=\frac{\sin ^{2}[\pi(\alpha-m)]}{[\pi(\alpha-m)]^{2}},
$$

where $m$ is the diffraction order and $\alpha$ is the detuning parameter:

$$
\alpha=\frac{\lambda_{0}}{\lambda} \frac{n(\lambda)-1}{n\left(\lambda_{0}\right)-1},
$$

$\lambda_{0}$ is the designed wavelength, $n$ is the refractive index. The calculated diffraction efficiency for diffractive optical element working in spectral range $200 \mathrm{~nm}-300 \mathrm{~nm}$ is shown in Fig. 5. The central wavelength was chosen to be equal to $236 \mathrm{~nm}$, in that way one obtains almost equal efficiencies at the extreme wavelengths.

To overcome the problem of the drop in diffraction efficiency in broad spectral range, multilayer diffractive optical elements have been proposed $[9,10]$. They consist of at least two harmonic diffractive structures with the same zone widths but manufactured in different materials. The two structures may be in contact with each other or be placed very close to each other with the gap at the level of several wavelengths. In the latter case one gets additional degree of freedom in the design the depths of the two profiles can be made different. For a MLDOE made of two diffractive structures it is possible to obtain maximal theoretical diffraction efficiency for two chosen wavelengths and significantly higher average efficiency in the whole spectrum compared to SLDOEs. Such solutions are used in some commercially

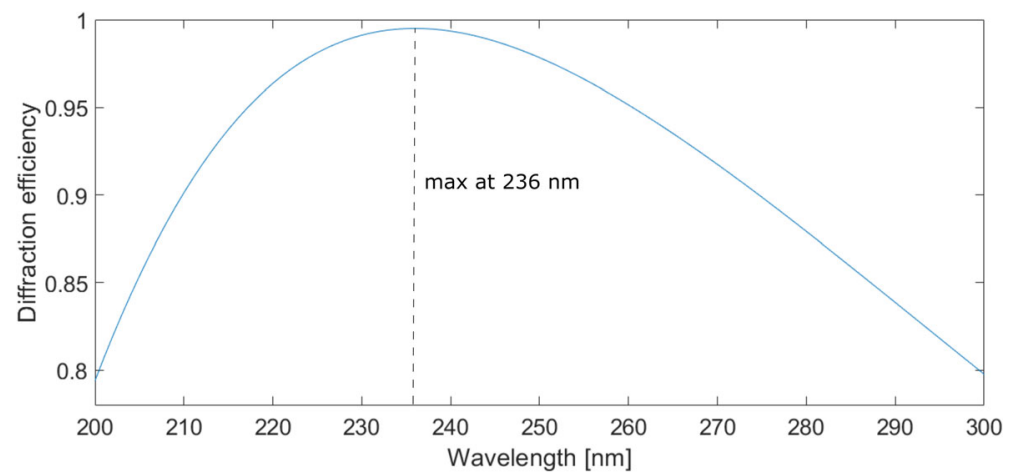

Fig. 5 Diffraction efficiency of SLDOE in spectral range 200-300 nm 
available photo objectives [11], where diffractive structures are made in ultraviolet curable resins. However, using MLDOEs comes at the price of increased complexity. First of all, the structures have to be accurately positioned with respect to each other, both laterally and axially. Moreover, harmonic diffractive structures are significantly deeper than SLDOEs. Due this fact some standard manufacturing methods may not be used. Additionally, when the ratio of zone width to the profile depth is too small, below approximately 10 , scalar approximation is no longer valid and consequently real diffraction efficiency of the element may be lower than calculated by the formulas derived based on those approximations [12].

In the case of designed hybrid telescopes, assuming that fused silica and magnesium fluoride as the materials for MLDOE, the depths of the profiles would need to be equal to $4 \mu \mathrm{m}$ and $5.7 \mu \mathrm{m}$, calculated based on the formulas presented by Dun et al. [13]. Comparing it to the minimal zone widths of the required elements of $22.6 \mu \mathrm{m}$, one can see that the structures may no longer be considered to be planar. Additionally, since the diffractive element in the system is illuminated under certain range of angles, a significant shielding effect may occur, leading to a further decrease of diffraction efficiency [14]. Due to these difficulties it was decided that in this project using SLDOE is more beneficial. In order to eliminate the problem of the strong dependence of the diffraction efficiency on wavelength it is considered to use a SLDOE with varying profile depth. It is possible to design an element in which different zones have profile depth matched to different wavelength and by proper optimization equal diffraction efficiency in the whole spectral range may be obtained $[15,16]$.

Energy diffracted to higher orders will form strongly defocused images of the observed objects. If the designed order is -1 , even the nearest orders -2 and 0 will create spots larger than $1 \mathrm{~mm}$ in diameter. As a consequence, for a single point-like object, energy density on the detector coming from those other diffractive orders will be below detector sensitivity. However, considering that there may be a significant number of observable objects at the same time, those defocused images of different objects may overlap and as a result create a detectable background. Figure 6 shows the expected performance of the hybrid system with included average diffraction efficiency of the DOE equal to $91 \%$. Presented curves were obtained by scaling the values from Fig. 4 c) and d) by 0.91 , based on the model described by Wang et al. [17].

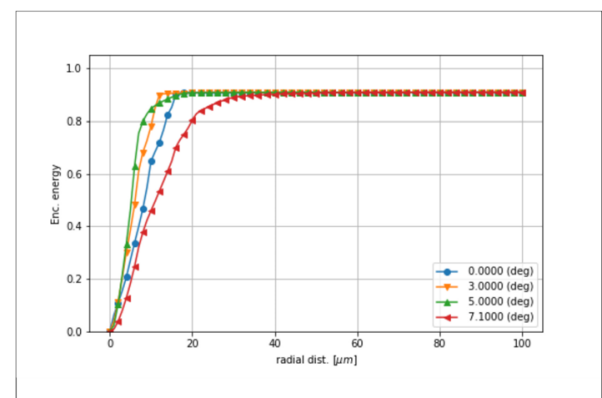

a)

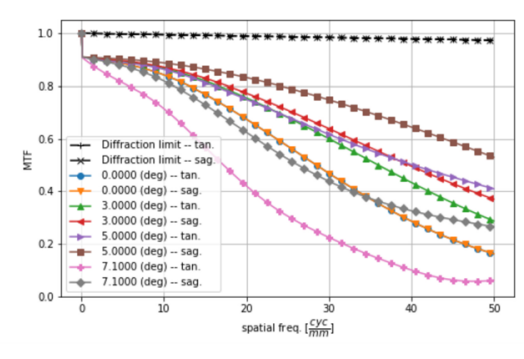

b)

Fig. 6 Scaled performance plots for hybrid refractive-diffractive system. a) geometric encircled energy, b) MTF curves 
Although a negative influence of the higher diffraction orders is visible, performance of the system is still similar to the reflective telescope despite having significantly larger aperture. Additionally, due to the fact that the image will consist of a certain number of bright stars images on a dark background, this blur should not lead to the loss of details and the influence of the higher diffraction orders could be easily decreased. Methods like deconvolution using modelled point spread function of the system have been proposed $[15,18]$.

\section{Conclusions}

In this paper a design of a hybrid refractive-diffractive telescope with a $90 \mathrm{~mm}$ entrance pupil diameter working in the spectral range $200 \mathrm{~nm}-300 \mathrm{~nm}$ and with large field of view $10^{\circ} \times 10^{\circ}$ is presented. It offers significantly improved imaging quality compared to a purely refractive system with similar parameters and one more element. Its performance is also compared to a reflective system with the same field of view and a smaller entrance pupil diameter equal to $50 \mathrm{~mm}$. The hybrid one offers similar aberration correction while having significantly lower f-number, as well as significant reduction in size, making it more suitable for use as a payload of microsatellites.

Two types of diffractive optical elements used in imaging systems are presented. In this particular design it is more advantageous to use the simpler one - a single layer diffractive optical element. By optimization of the DOE's profile depth it is possible to obtain uniform diffraction efficiency for the whole required spectral range. The undesired influence of non-imaging diffractive orders may be removed by subsequent image processing.

Acknowledgements This work has been carried out as a part of cooperation between Solaris Optics S.A. and Warsaw University of Technology in the frame of the Implementation Doctorate program, agreement no. 15/DW/2017/01/1, under the supervision of Jerzy Krężel, PhD, R\&D Manager at Solaris Optics S.A., and Michał Józwik, PhD, Associate Professor at the Faculty of Mechatronics, Warsaw University of Technology. Described system is a potential payload for a mission which concept has been presented in 'Astronomical Observation Satellite in UV; Pre-feasibility study for UVSat satellite', 2016, Polish Space Agency, Warsaw.

Funding information This work was partially funded by the Polish Ministry of Science and Higher Education in the frame of the Implementation Doctorate program, agreement no. 15/DW/2017/01/1.

\section{Compliance with ethical standards}

Conflict of interest The author declares that he has no conflict of interest.

Open Access This article is licensed under a Creative Commons Attribution 4.0 International License, which permits use, sharing, adaptation, distribution and reproduction in any medium or format, as long as you give appropriate credit to the original author(s) and the source, provide a link to the Creative Commons licence, and indicate if changes were made. The images or other third party material in this article are included in the article's Creative Commons licence, unless indicated otherwise in a credit line to the material. If material is not included in the article's Creative Commons licence and your intended use is not permitted by statutory regulation or exceeds the permitted use, you will need to obtain permission directly from the copyright holder. To view a copy of this licence, visit http://creativecommons.org/licenses/by/4.0/. 


\section{References}

1. Sachkov M., Shustov B., and Gómez de Castro A. I.: Instrumentation of the WSO-UV project, Proc. SPIE 9144, id. 914402 (2014); https://doi.org/10.1117/12.2055513;

2. Tandon, S.N., Hutchings, J.B., Ghosh, S.K., et al.: In-orbit performance of UVIT and first results. J. Astrophys. Astron. 38, 28 (2017). https://doi.org/10.1007/s12036-017-9445-x;

3. Roberge A., Bolcar M. R., France K. C.: Telling the story of life in the cosmos: the LUVOIR telescope concepts, Proc. SPIE 11115, id. 111150 O (2019), https://doi.org/10.1117/12.2530475;

4. Wong I., Brown M. E., Blacksberg J., Ehlmann B. I., Mahjoub A.: Hubble Ultraviolet Spectroscopy of Jupiter Trojans, The Astronomical Journal, Volume 157, Issue 4, article id. 161, 7 pp. (2019), https://doi. org/10.3847/1538-3881/ab0e00;

5. Pigulski, A., et al.: UVSat: a concept of an ultraviolet/optical photometric satellite. PTA Proceedings. 123, 51-56 (2013)

6. Johnson J.: Selection of Materials for UV Optics, https://pdfs.semanticscholar.org/10a5/6f27a2e2455 cf531ccb24d617dbe651139d6.pdf (2008). Accessed 11 December 2019;

7. O'Shea D. C. et al.: Diffractive Optics. Design, Fabrication, and Test, Chapter 4 "Diffractive Lens Design", p. 57-82, ISBN 0-8194-5171-1, SPIE Press (2004)

8. Hallam K. L., Howell B. J., Wilson M. E.: Wide-angle flat field telescope, United States Patent 4,598, 981, issued 8 July 1986;

9. Choi J., Cruz-Cabrera A. A., Tanbakuchi A.: Practical implementation of broadband diffractive optical elements, Proc. SPIE 8612, Micromachining and Microfabrication Process Technology XVIII, 86120G (9 March 2013); https://doi.org/10.1117/12.2013204;

10. Xue, C., Cui, Q.: Design of multilayer diffractive optical elements with polychromatic integral diffraction efficiency. Opt. Lett. 35, 986-988 (2010)

11. Nakai T., Ogawa H.: Research on multi-layer diffractive optical elements and their application to camera lenses, in Diffractive Optics and Micro-Optics, R. Magnusson, ed., Vol. 75 of OSA Trends in Optics and Photonics Series (Optical Society of America, 2002), paper DMA2, https://doi.org/10.1364 /DOMO.2002.DMA2;

12. Huo, F., Wang, W., Xue, C.: Limits of scalar diffraction theory for multilayer diffractive optical elements. Optik. 127, 5688-5694 (2016)

13. Dun, X., Jin, W., Wang, X.: Material selection and corresponding optimal surface relief height for multilayer diffractive optical elements. Opt. Eng. 54(11), 115105 (2015). https://doi.org/10.1117/1. OE.54.11.115105;

14. Yang, H., Xue, C.: Influence of passive facet of multilayer diffractive optical elements. Appl. Opt. 57, 2604-2609 (2018). https://doi.org/10.1364/AO.57.002604

15. Peng Y., Fu Q., Heide F., Heidrich W.: The Diffractive Achromat Full Spectrum Computational Imaging with Diffractive Optics, SIGGRAPH '16 Technical Paper, Anahaim, CA, ISBN 978-1-4503-4279-7/16/ 07, (24-28 July 2016), https://doi.org/10.1145/2897824.2925941;

16. Pietarinen, J., Vallius, T., Turunen, J.: Wideband four-level transmission gratings with flattened spectral efficiency. Opt. Express. 14, 2583-2588 (2006). https://doi.org/10.1364/OE.14.002583;

17. Wang, T., et al.: Evaluation of the imaging performance of hybrid refractive-diffractive systems using the modified phase function model. J. Opt. 12, 045705 (2010). https://doi.org/10.1088/2040-8978/12 /4/045705

18. Hu, Y., Cui, Q., Zhao, L., Piao, M.: PSF model for diffractive optical elements with improved imaging performance in dual-waveband infrared systems. Opt. Express. 26, 26845-26857 (2018). https://doi. org/10.1364/OE.26.026845;

Publisher's note Springer Nature remains neutral with regard to jurisdictional claims in published maps and institutional affiliations. 